\title{
TORTA DE FILTRO E TURFA NA MITIGAÇÃO DE SOLO CONTAMINADO COM RESÍDUO DE SUCATA RICO EM BORO (1)
}

\author{
RICARDO ALEXANDRE BRASIL JORGE ( $\left.{ }^{2}\right)$; CLEIDE APARECIDA DE ABREU $\left(3^{*}\right)$; \\ CRISTIANO ALBERTO DE ANDRADE $\left({ }^{3}\right)$; OTÁVIO ANTONIO DE CAMARGO $\left({ }^{3,4}\right)$
}

\begin{abstract}
RESUMO
O objetivo deste trabalho foi avaliar a torta de filtro e a turfa como fontes de matéria orgânica para mitigar solo contaminado com boro, sob o cultivo do nabo forrageiro (Raphanus sativus). O solo da área estudada foi contaminado pelo descarte de resíduo metálico rico em B, com teor total de $62,1 \mathrm{mg} \mathrm{kg}^{-1}$ e disponível (água quente) de $8,0 \mathrm{mg} \mathrm{dm}^{-3}$. O solo recebeu aplicação de $10 \mathrm{t} \mathrm{ha}^{-1}$ de calcário por recomendação do órgão ambiental do Estado de São Paulo, tendo o valor de $\mathrm{pH}$ elevado a 7,5. O delineamento experimental foi o inteiramente casualizado em esquema fatorial $2 \times 4$, com três repetições. As doses de cada material orgânico adicionado fundamentaram-se nos teores de C, sendo equivalentes a 0, 20, 40 e $80 \mathrm{t} \mathrm{ha}^{-1}$. Em cada parcela experimental foram utilizados vasos contendo 5,0 kg de solo contaminado, cultivado com dez plantas de nabo forrageiro. Não se constataram nas plantas sintomas de toxicidade, embora teores elevados tenham sido observados no caule + folhas $\left(367\right.$ a $646 \mathrm{mg} \mathrm{kg}^{-1}$ ) e na vagem $\left(119\right.$ a $\left.180 \mathrm{mg} \mathrm{kg}^{-1}\right)$. De acordo com o fator de transferência, índice de translocação e do tempo de remoção, seriam necessários, para remover $50 \%$ do teor de B total do solo, 10,6 anos sem uso de MO; 15,4 anos com uso da turfa; 9,3 anos com uso da torta de filtro. Concluiu-se que a torta de filtro foi considerada mitigadora para o solo contaminado e que o nabo forrageiro tem potencial para ser cultivado na área contaminada.
\end{abstract}

Palavras-chave: Raphanus sativus, matéria orgânica, remediação de solos.

\section{ABSTRACT \\ FILTER CAKE AND PEAT AS AMENDMENTS OF CONTAMINATED SOIL WITH RESIDUE OF SCRAP RICH IN BORON}

The objective of this study was to evaluate the filter cake and peat as sources of organic matter in order to mitigate contaminated soil with boron, under the cultivation of turnip (Raphanus sativus). The studied soil was contaminated by the disposal of metallic waste rich in B which increased the total content of $B\left(62.1 \mathrm{mg} \mathrm{kg}^{-1}\right)$, and its availability $\left(8.0 \mathrm{mg} \mathrm{dm}^{-3}\right)$. The soil received application of $10 \mathrm{t} \mathrm{ha}^{-1}$ of limestone as recommendation of the environmental agency of the State of São Paulo, and the $\mathrm{pH}$ value increased to 7.5. The experimental design was completely randomized in a factorial scheme $2 \times 4$, with three replicates. The doses of each organic material added were based on the content of $C$, being equivalent to $0,20,40$ and $80 \mathrm{t} \mathrm{ha}^{-1}$. In each experimental plot, pots containing $5.0 \mathrm{~kg}$ of contaminated soil was cultivated with ten plants each of turnip. The plants showed no symptoms of toxicity, although high levels have been observed in the leaf + stem (367 to $646 \mathrm{mg} \mathrm{kg}^{-1}$ ), and pod (119 to $180 \mathrm{mg} \mathrm{kg}^{-1}$ ). The indices $\mathrm{F}$, IT, and the removal indicated the feasibility of phytoextraction of B by turnip. According to these indices, to remove $50 \%$ of the total B content of soil, it would be necessary 10.6 years without using OM, 15.4 years using peat, and 9.3 years using filter cake. It was concluded that the filter cake was considered amendment to the contaminated soil, and that the turnip has the potential to be grown in the contaminated area.

Key words: Raphanus sativus, organic matter, remediation of soils.

( $\left.{ }^{1}\right)$ Recebido para publicação em 4 de setembro de 2009 e aceito em 22 de dezembro de 2009.

$\left({ }^{2}\right)$ Aluno de Mestrado no Curso de Agricultura Tropical e Subtropical, área de Concentração Gestão de Recursos Agroambientais, Instituto Agronômico (IAC), Campinas, Caixa Postal 28, 13012-970 Campinas (SP). E-mail: r.brasil@petrobras.com.br

$\left({ }^{3}\right)$ Centro de Pesquisa e Desenvolvimento em Solos e Recursos Ambientais, Instituto Agronômico - IAC, Campinas (SP). E-mail: cleide@iac.sp.gov.br $\left(^{*}\right)$ Autora correspondente; andrade@iac.sp.gov.br; ocamargo@iac.sp.gov.br

$\left({ }^{4}\right)$ Pesquisador do CNPq. 


\section{INTRODUÇÃO}

A CETESB estabeleceu valores orientadores de diversos elementos para solos do Estado de São Paulo, mas não fez referência ao boro (CЕTESв, 2009). Até na legislação internacional, os valores dos limites máximos de B em solo são escassos. A legislação austríaca considera o teor total de $100 \mathrm{mg} \mathrm{kg}^{-1}$ como o limite máximo para B em solos (Kabata-Pendias, 2001).

Na literatura há poucos relatos quanto à relação entre concentração de $B$ total no solo e toxicidade em plantas, sendo mais utilizados valores de B disponível. Abreu et al. (2005) citam que concentrações de B disponível acima de 3,0 $\mathrm{mg} \mathrm{dm}^{-3}$ podem ser tóxicas para a maioria das plantas.

O B está intrinsecamente ligado aos minerais do solo (Kabata-Pendias, 2001), tendo a disponibilidade governada pelos processos de adsorção e dessorção que, por sua vez, são influenciados pelo $\mathrm{pH}$ e matéria orgânica do solo (Alleoni e Camargo, 2000; Sharma et al., 2006). Nos valores de $\mathrm{pH}$ entre 5,0 e 7,0 há maior disponibilidade de $\mathrm{B}$ no solo, enquanto na faixa de pH entre 7,0 e 9,0 ocorre maior adsorção desse elemento (LindSAY, 1979). Portanto, a calagem é uma das práticas mais eficientes na minimização da disponibilidade de $\mathrm{B}$.

Outro agente mitigador de solos contaminados por B é a matéria orgânica, que pode reduzir a disponibilidade desse elemento e, consequentemente, o efeito tóxico às plantas (Kumpiene et al., 2008). Esse processo ocorre pelo fato de a matéria orgânica ser um importante ligante, tanto para cátions metálicos como para ânions inorgânicos, complexando e imobilizando esses elementos, característica que é atribuída aos ácidos orgânicos (SchWAB et al., 2007).

Aliadas à aplicação de materiais mitigadores de solos contaminados, têm-se usado espécies de plantas que são capazes de absorver e acumular na parte aérea altos teores do contaminante (RASKIN e Ensley, 2000; Usepa, 2009a). Essas espécies são chamadas de plantas fitoextratoras, que, além das características anteriormente mencionadas, devem ter alta produção de biomassa (MARTínEz et al., 2006). Algumas das características das fitoextratoras têm sido reconhecidas em plantas da família Brassicaceae (BRoоKs, 2004). O nabo forrageiro (Raphanus sativus), pertencente a essa família, é citado entre as muitas plantas fitoextratoras de metais pesados e boro (BAÑuelos et al., 1993; SANTOS et al., 2008).

Existem algumas maneiras de avaliar o potencial de plantas para fitoextração de uma espécie química qualquer, como o uso do fator de transferência (F), que avalia a transferência do elemento de interesse do solo para a planta, e o índice de translocação (IT), que avalia a translocação desse elemento da raiz para a parte aérea (Bose e BHATtAcharyya, 2008). Além dos fatores auxiliarem na avaliação da planta para uso na fitorremediação de áreas contaminadas, também permitem estimar o tempo necessário para reduzir as concentrações tóxicas do(s) elemento(s) de interesse.

A proposta deste trabalho foi avaliar o potencial do nabo forrageiro em tolerar e acumular níveis elevados de boro em solo multicontaminado e também o uso da torta de filtro e da turfa como mitigadores desse elemento no solo.

\section{MATERIAL E MÉTODOS}

\subsection{Caracterização do solo}

A pesquisa foi realizada com amostra de solo, classificado como Neossolo Litólico Chernossólico fragmentário, textura média e imperfeitamente drenado (EMBRAPA, 2006). O local de coleta desse solo, com área de aproximadamente 3 ha, foi contaminado em razão de descarte de resíduo de sucata metálica rica em boro. O B está associado às ligas metálicas, tendo ampla aplicabilidade na metalurgia (SOAREs et al., 2005). A área foi interditada pelo órgão ambiental e, logo após a interdição, foi feita a aplicação de $10 \mathrm{t}$ $\mathrm{ha}^{-1}$ de calcário dolomítico ao solo, elevando o seu $\mathrm{pH}$ para 7,5.

A amostra de solo foi coletada na camada de 0$0,20 \mathrm{~m}$ de profundidade, seca ao ar e peneirada em peneira de malha de $2,0 \mathrm{~mm}$. O teor de $\mathrm{B}$ total original, determinado de acordo com método da UsEPA 3051, conforme descrito em UsEPA (2009b), foi igual a 62,1 mg $\mathrm{kg}^{-1}$. Algumas características químicas do solo, determinadas conforme descrito em RAIJ et al. (2001), foram: $\mathrm{pH}_{\mathrm{CaCl} 2}=7,5 ; \mathrm{MO}=30,5 \mathrm{~g} \mathrm{dm}^{-3} ; \mathrm{P}=43,3 \mathrm{mg} \mathrm{dm}^{-3}$; $\mathrm{K}=2,6 \mathrm{mmol}_{\mathrm{c}} \mathrm{dm}^{-3} ; \mathrm{Ca}=294 \mathrm{mmol}_{\mathrm{c}} \mathrm{dm}^{-3} ; \mathrm{Mg}=59 \mathrm{mmol}_{\mathrm{c}} \mathrm{dm}^{-3}$; $\mathrm{CTC}=364 \mathrm{mmol}_{\mathrm{C}} \mathrm{dm}^{-3} ; \mathrm{H}+\mathrm{Al}=9,0 \mathrm{mmol}_{\mathrm{C}} \mathrm{dm}^{-3} ; \mathrm{V}=98 \%$; $\mathrm{B}=8,5 \mathrm{mg} \mathrm{dm}^{-3} ; \mathrm{Cu}=61,6 \mathrm{mg} \mathrm{dm}^{-3}, \mathrm{Fe}=15,5 \mathrm{mg} \mathrm{dm}^{-3}$, $\mathrm{Mn}=12,0 \mathrm{mg} \mathrm{dm}^{-3} \mathrm{e} \mathrm{Zn}=452 \mathrm{mg} \mathrm{dm}^{-3}$.

\subsection{Material orgânico e incubação com o solo}

Como fontes de matéria orgânica foram utilizadas a torta de filtro e a turfa (Tabela 1). A torta de filtro é um resíduo proveniente do tratamento do caldo de cana-de-açúcar na indústria açucareira. A turfa é um material orgânico natural, estabilizado e reconhecido por sua alta capacidade de troca iônica. 
Para o cálculo das doses utilizadas, foi considerado o teor de C orgânico de cada material (Tabela 1), sendo as quantidades adicionadas ao solo equivalentes a: $0 ; 20 ; 40$ e $80 \mathrm{t} \mathrm{ha}^{-1} \mathrm{de} \mathrm{C}$. Esses valores foram, em $\mathrm{g} \mathrm{kg}^{-1}$, iguais a: $0 ; 37,9 ; 75,8$ e 151,6 para a torta de filtro e de $0 ; 61,3 ; 122,6 ; 245,2$ para a turfa. Após a adição dos materiais orgânicos ao solo, foi feita mistura e homogeneização, mantendo-se a umidade do solo em $60 \%$ da capacidade de retenção. O solo foi incubado, em seguida, durante 35 dias. Aos 20 dias de incubação, promoveu-se a adição de $\mathrm{P}$ em dose equivalente a $200 \mathrm{mg} \mathrm{kg}^{-1}$, na forma de superfosfato triplo, seguindo-se de homogeneização e incubação por mais 15 dias.

Tabela 1. Valores de $\mathrm{pH}$, condutividade elétrica, teor de $\mathrm{C}$ orgânico, relação $\mathrm{C} / \mathrm{N}$ e alguns nutrientes disponíveis nos materiais orgânicos adicionados ao solo

\begin{tabular}{|c|c|c|c|c|c|c|c|c|c|c|c|}
\hline Fonte de $\mathrm{C}_{\text {org }}$ & $\mathrm{pH}$ & $\mathrm{EC}$ & C. org & $\mathrm{C}: \mathrm{N}$ & $\mathrm{N}-\mathrm{NO}_{3}^{-}$ & $\mathrm{NH}_{4}$ & $\mathrm{P}$ & $\mathrm{K}$ & $\mathrm{Ca}$ & $\mathrm{Mg}$ & B \\
\hline & & $\mathrm{dS} \mathrm{m}^{-1}$ & $\mathrm{~g} \mathrm{~kg}^{-1}$ & & & & 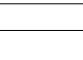 & $\mathrm{ng} \mathrm{L}^{-}$ & & & \\
\hline Turfa & 5,5 & 0,2 & 163,1 & 24,3 & 8,6 & 1,8 & 0,1 & 2,9 & 5,8 & 3,9 & 0,02 \\
\hline Torta & 7,5 & 0,9 & 263,7 & 12,0 & 3,5 & 61,4 & 10,1 & 37,0 & 70,8 & 52,0 & $<0,01$ \\
\hline
\end{tabular}

Os nutrientes disponíveis foram determinados pelo método da extração 1:1,5 descrito por SonNEveld et al. (1974)

\subsection{Manejo do experimento e análises químicas da planta e do solo}

O experimento foi desenvolvido em casa de vegetação, em delineamento experimental inteiramente casualizado e esquema fatorial $2 x$ 4, usando-se três repetições. Cada parcela experimental foi constituída de vaso contendo 5,0 $\mathrm{kg}$ de solo, com dez plantas de nabo forrageiro (Raphanus sativus).

Durante todo o período de manejo do experimento, a umidade do solo foi mantida a $60 \%$ de sua capacidade máxima de retenção. Todas as parcelas receberam adubação nitrogenada em dose igual a $30 \mathrm{mg} \mathrm{kg}^{-1}$ de $\mathrm{N}$, na forma de nitrato de amônio, que foi parcelada em duas aplicações, metade por ocasião da emergência das plântulas e metade, 15 dias depois. O período de duração do experimento foi de dezembro de 2007 a fevereiro de 2008. O corte do nabo forrageiro foi realizado após 65 dias da semeadura, separando-se caule + folha, vagem e raízes.

O material vegetal da parte aérea (folhas, caule e vagem) foi lavado em água corrente, seguida de uma solução a $1 \%$ de $\mathrm{HCl}$ e água destilada. Após lavagem, o material vegetal foi colocado em estufa para secagem a $70{ }^{\circ} \mathrm{C}$ e, depois de seco, foi pesado e moído em moinho do tipo Wiley. As raízes do nabo forrageiro foram separadas da terra por peneiramento, lavadas em água corrente, imersas por 90 min em solução $0,02 \mathrm{mmol} \mathrm{L}^{-1}$ de EDTA dissódico e, finalmente, lavadas em água destilada. Os procedimentos de secagem e moagem foram idênticos aos da parte aérea. Todo o material vegetal foi submetido à digestão com
$\mathrm{HNO}_{3} / \mathrm{H}_{2} \mathrm{O}_{2}$, em forno de micro-ondas (ABReu, 1997), para posterior determinação dos teores de macro e micronutrientes usando-se a espectrometria de emissão por plasma ICP-OES, com exceção do K, que foi por fotometria de chama.

Nas amostras de solo coletadas após o período de incubação e antes da semeadura, foram determinados o teor total de $\mathrm{B}$, pelo extrator $\mathrm{HNO}_{3}$, de acordo com o método da UsEPA 3051 (UsEPA, 2009b) e o teor disponível, usando-se água quente e azometina - $\mathrm{H}$, aquecimento em forno de micro-ondas, conforme descrito em ABREU et al. (2001). Os teores disponíveis de $\mathrm{P}, \mathrm{K}, \mathrm{Ca}$ e $\mathrm{Mg}$ foram extraídos pela resina trocadora (RAIJ et al., 2001).

\subsection{Análise estatística}

Os resultados foram submetidos a análise de variância (ANOVA) e no caso do teste $\mathrm{F}$ significativo (ANOVA) foi aplicado teste de Tukey a $5 \%$ para comparação de médias entre os materiais orgânicos e a análise de regressão para doses (modelos linear e quadrático). No caso de interação significativa entre os fatores estudados, fez-se o desdobramento. As análises estatísticas foram realizadas usando o software SISVAR 5.0 (FERREIRA, 1999).

\subsection{Avaliação da transferência e remoção de boro}

A transferência de B do solo para a planta foi avaliada pelo fator de transferência $(\mathrm{F})$, adaptado de LUBben e SAUERBECK (1991), pela equação: $\mathrm{F}=\mathrm{CPT} / \mathrm{CT}$, sendo CPT a concentração de B na planta toda (mg $\left.\mathrm{kg}^{-1}\right)$ e CT a concentração total de B no solo $\left(\mathrm{mg} \mathrm{kg}^{-1}\right)$. 
A translocação do B da raiz para a parte aérea foi avaliada por meio do índice de translocação (IT\%), conforme sugerido por ABICHEQUER e BOHRLEN (1998), pela equação: IT\% $=(\mathrm{QPA} / \mathrm{QAP}) \times 100$, sendo QPA a quantidade acumulada de $B$ na parte aérea $\left(\mathrm{mg}\right.$ vaso $^{-}$ ${ }^{1}$ ) e QAP a quantidade acumulada de B na planta toda $\left(\mathrm{mg} \mathrm{vaso}^{-1}\right)$.

A partir desses índices foi determinada a eficiência de remoção (E\%) de $B$, dado pela equação: $\mathrm{E} \%=(\mathrm{QPA} / \mathrm{QR}) \times 100$, sendo $\mathrm{QPA}$ a quantidade acumulada de $B$ na parte aérea $\left(\mathrm{mg}\right.$ vaso $\left.^{-1}\right)$ e $Q R$ a quantidade acumulada total de $\mathrm{B}$ a ser extraído do solo $\left(\mathrm{mg}\right.$ vaso $\left.^{-1}\right)$.

O tempo de remoção, em anos, necessário para reduzir em $50 \%$ o teor de $\mathrm{B}$ do solo, é dado pela fórmula: $\mathrm{T}=(\mathrm{R} / \mathrm{E} \%) / \mathrm{NC}$; sendo $\mathrm{R}$ a porcentagem de $\mathrm{B}$ a ser removida do solo, $\mathrm{E} \%$ é a eficiência de remoção e NC é o número de ciclos da cultura. $\mathrm{O}$ percentual de remoção (R) de B foi arbitrado em $50 \%$, embora não haja valor de referência para este elemento definido pela Cетеsв. Nestes cálculos, considerou-se apenas um ciclo de cultivo por ano do nabo forrageiro, visto que a planta é considerada cobertura de inverno.

\section{RESULTADOS E DISCUSSÃO}

\subsection{Teor total e disponível de boro no solo e outros atributos químicos}

No solo estudado havia teor total de B igual a $62,1 \mathrm{mg} \mathrm{kg}^{-1}$, valor este dentro da faixa citada por BISSANI et al. (2008) como de ocorrência natural, que varia de 7 a $80 \mathrm{mg} \mathrm{kg}^{-1}$. Para solos tropicais, teores iguais a esse são considerados elevados (TANAKA E FujIWARA, 2008). Em São Paulo, a CETESB não estabelece valores orientadores para o $\mathrm{B}$ no solo e limites máximos para esse elemento são escassos, mesmo considerando a legislação internacional. De acordo com KaвATAPendias (2001), há na legislação austríaca um limite máximo para B total em solos, cujo valor é de $100 \mathrm{mg}$ $\mathrm{kg}^{-1}$. Portanto, a concentração total de B no solo estudado está abaixo de alguns limites máximos citados na literatura, embora esse fato não seja sinônimo de ausência de impacto negativo no crescimento de plantas.

O teor total de B no solo não foi influenciado pela adição dos materiais orgânicos, o que era esperado em razão do baixo aporte de B via torta de filtro e turfa (Tabela 1). O teor total médio de B no solo foi de $65,1 \mathrm{mg} \mathrm{kg}^{-1}$ pela adição de torta de filtro e de $60,1 \mathrm{mg} \mathrm{kg}^{-1}$, em virtude da aplicação de turfa, conforme tabela 2 .
Quanto aos teores disponíveis, houve acréscimo para B, P, K e Ca com a aplicação de torta de filtro (Tabela 2). Com esse material orgânico, o teor médio de $B$ disponível aumentou $0,8 \mathrm{mg} \mathrm{dm}^{-3}$, representando um incremento de $10 \%$. Este aumento foi expressivo, considerando-se a faixa de teor adequado de $B$ em solos entre 1,2 a 3,0 $\mathrm{mg} \mathrm{dm}^{-3}$ para a maioria das culturas (ABREU et al., 2005). O aumento na disponibilidade de alguns nutrientes pelo uso de torta de filtro pode ser explicado com relação à sua composição e à qualidade do material. Segundo Espinoza e Oliveira (1984), a torta de filtro é um material considerado rico em N, P e Ca e, portanto, deve ter contribuído para o aumento desses nutrientes no solo. Além disso, a torta de filtro, por ter a mais baixa relação $\mathrm{C} / \mathrm{N}$ (Tabela 1) em comparação com a turfa, é mais facilmente degradada, disponibilizando os nutrientes complexados no material orgânico. A solubilização de nutrientes em materiais orgânicos é comumente relacionada com a presença de ácidos fúlvicos em maiores proporções que ácidos húmicos (SAntos et al., 2008); para biossólidos frescos, por exemplo, com menor grau de humificação, há mais ácidos fúlvicos que húmicos (Kumpiene et al., 2008). Por outro lado, na turfa há predominância de ácidos húmicos em relação aos ácidos fúlvicos, em função do maior tempo necessário para a sua formação e da sua maior estabilidade orgânica, conforme observado no trabalho de SYROVETNIK (2005).

Efeitos tóxicos em plantas têm sido mais relacionados com teores disponíveis que com teores totais no solo. Tanto na adição de torta de filtro como na de turfa, o B disponível variou de 7,7 a $8,8 \mathrm{mg} \mathrm{dm}^{-3}$.

AzARENCO (2007) constatou $4 \%$ de perda de produtividade de alfafa (Medicago sativa) cultivada em solo cujo B disponível era de 7,0 a $8,0 \mathrm{mg} \mathrm{kg}^{-1}$. Tal perda aumentou para $31 \%$ quando o $\mathrm{B}$ disponível foi de 17,5 a $18,9 \mathrm{mg} \mathrm{kg}^{-1}$. A toxicidade de B afeta a produtividade pela diminuição da absorção de água e nutrientes pelas raízes, afetando o crescimento e desenvolvimento das plantas (FujIWARA e TANAKA, 2008; Кот, 2009).

$\mathrm{O}$ pH do solo permaneceu próximo a 7,5, independentemente da aplicação dos tratamentos (Tabela 2). Lehto (1995) observou que a adsorção de B pelo solo foi linear quando se elevou o $\mathrm{pH}$ do solo orgânico de 3,5 para 5,9 por meio da calagem. Se, por um lado, o aumento do $\mathrm{pH}$ implica em menor remoção do B pelas plantas e, portanto, menor eficiência da fitoextração, a reação alcalina do solo também contribui para a redução de possíveis efeitos tóxicos às plantas, possibilitando seu estabelecimento na área contaminada e início do processo de fitorremediação. 
Tabela 2. Efeito da aplicação de doses de torta de filtro e turfa no solo, nos valores de $\mathrm{pH}$ em $\mathrm{CaCl}_{2}$, disponíveis de $\mathrm{P}$, $\mathrm{K}, \mathrm{Ca}, \mathrm{Mg}, \mathrm{B}$, e B total

\begin{tabular}{|c|c|c|c|c|c|c|c|c|}
\hline \multirow{2}{*}{ Tratamento } & \multirow{2}{*}{$\begin{array}{l}\text { Dose } \\
\text { Corg }_{\text {org }}\end{array}$} & \multirow{2}{*}{$\begin{array}{c}\mathrm{pH} \\
\mathrm{CaCl}_{2}\end{array}$} & $\mathrm{P}$ & $\mathrm{K}$ & $\mathrm{Ca}$ & $\mathrm{Mg}$ & \multirow{2}{*}{$\begin{array}{c}\text { B } \\
\text { água quente }\end{array}$} & \multirow{2}{*}{$\begin{array}{c}\text { B } \\
\text { USEPA }\end{array}$} \\
\hline & & & \multicolumn{4}{|c|}{ resina } & & \\
\hline & $\mathrm{tha}^{-1}$ & & $\mathrm{mg} \mathrm{dm}^{-3}$ & 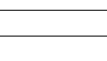 & $\mathrm{mol}_{\mathrm{c}} \mathrm{dr}$ & & $\mathrm{mg} \mathrm{dm}^{-3}$ & $\mathrm{mg} \mathrm{kg}^{-3}$ \\
\hline \multirow[t]{5}{*}{ Turfa } & 0 & 7,6 & $45 b$ & 2,4 & $290 \mathrm{~b}$ & 57 & 8,1 & 65,8 \\
\hline & 20 & 7,6 & $41 b$ & 2,3 & $226 b$ & 45 & 8,0 & 60,8 \\
\hline & 40 & 7,5 & $42 b$ & 2,4 & $246 b$ & 52 & 7,9 & 57,5 \\
\hline & 80 & 7,4 & $36 b$ & 2,2 & $183 b$ & 50 & 7,7 & 56,2 \\
\hline & Média & 7,5 & $41 \mathrm{~B}$ & $2,3 \mathrm{~B}$ & $236 \mathrm{~B}$ & 51 & $7,9 \mathrm{~B}$ & 60,1 \\
\hline \multirow[t]{5}{*}{ Torta } & 0 & 7,4 & $42 b$ & 2,8 & $298 b$ & 61 & 8,0 & 58,4 \\
\hline & 20 & 7,6 & $83 a$ & 2,7 & $239 b$ & 47 & 8,8 & 55,7 \\
\hline & 40 & 7,5 & $125 a$ & 2,5 & $230 \mathrm{~b}$ & 53 & 8,5 & 75,5 \\
\hline & 80 & 7,6 & $176 a$ & 2,7 & $308 a$ & 56 & 8,6 & 70,9 \\
\hline & Média & 7,5 & $106 \mathrm{~A}$ & $2,7 \mathrm{~A}$ & $269 \mathrm{~A}$ & 54 & $8,7 \mathrm{~A}$ & 65,1 \\
\hline \multicolumn{9}{|l|}{$\overline{F V}$} \\
\hline Tipo M.O. & & ns & * & * & * & ns & * & $\mathrm{ns}$ \\
\hline Dose & & ns & * & ns & ns & ns & ns & ns \\
\hline M.O. x Dose & & $\mathrm{ns}$ & * & ns & * & ns & $\mathrm{ns}$ & ns \\
\hline
\end{tabular}

ANOVA: (ns) não significativo: (*) significativo; médias seguidas de letras diferentes na coluna diferem entre si ao nível de 5\% pelo teste de Tukey; letras minúsculas - comparação entre doses e letras maiúsculas - comparação entre tipos de material orgânico.

\subsection{Produção de massa seca pelo nabo forrageiro e concentração de nutrientes}

A produção de massa seca da parte aérea (caule, folhas e vagem) não foi afetada significativamente pelo tipo e dose do material orgânico, exceto para as raízes que apresentaram aumento de $25 \%$ na produção de massa seca, por causa da adição de torta de filtro (Tabela 3).

De modo geral, a pequena resposta na produção de massa seca do nabo forrageiro pode ser justificada, parcialmente, pela concentração adequada da maioria dos macronutrientes no caule + folhas (Tabela 4). Os valores médios para torta e turfa, em $\mathrm{g} \mathrm{kg}^{-1}$, foram respectivamente de: $\mathrm{P}=1,1$ e 2,$5 ; \mathrm{K}=$ 17,0 e 19,9; $\mathrm{Ca}=32,1$ e 38,5; $\mathrm{Mg}=3,7$ e 5,6. Estes valores, para essa planta, estão próximos aos anotados por CRUSCIOL et al. (2005) em cobertura de inverno, que obtiveram valores, em $\mathrm{g} \mathrm{kg}^{-1}$, de: $\mathrm{P}(5,3)$, $\mathrm{K}(19,2)$, Ca $(12,6)$ e Mg $(4,2)$.

A adição de doses de torta de filtro e turfa afetou, de maneira diferenciada, a concentração de macronutrientes e $B$ nas diversas partes da planta (Tabela 4). Nas raízes, não houve variabilidade significativa na concentração dos vários nutrientes; na vagem, a concentração foi pouco influenciada pelos tratamentos, destaque para Ca e B; e no caule + folhas, as concentrações de todos os nutrientes foram afetadas, sendo mais elevadas pela adição de torta de filtro (Tabela 4). A maior disponibilidade dos macronutrientes na torta de filtro (Tabela 1), bem como no solo tratado com esse material (Tabela 2) refletiuse em concentrações mais elevadas de P, K, Ca, Mg e B no compartimento caule + folhas, sendo essas, respectivamente, cerca de $130 \%, 20 \%, 20 \%, 50 \%$ e $40 \%$ superiores àquelas verificadas nas plantas dos tratamentos com turfa.

Comparando o teor de B nas várias partes da planta, que houve maior concentração no caule + folha, seguindo-se daquelas observadas na vagem e na raiz, fato justificado por sua translocação dentro da planta (Dordas et al., 2001).

Os teores de B observados, tanto no caule + folhas (367 a $646 \mathrm{mg} \mathrm{kg}^{-1}$ ) como na vagem (124 a 180 $\left.\mathrm{mg} \mathrm{kg}^{-1}\right)$, estavam altos para todos os tratamentos, inclusive nos controles (Tabela 4). SANTOs et al. (2008) relataram teor médio de $B$ na parte aérea do nabo forrageiro de $63 \mathrm{mg} \mathrm{kg}^{-1}$, em solo contendo $2,3 \mathrm{mg} \mathrm{dm}^{-3}$ de $B$ disponível. Cabe ressaltar que o teor de B disponível nesse solo era quatro vezes maior que o citado anteriormente. Na vagem, os teores estão muito acima daqueles relatados como adequados para 
Tabela 3. Produção de massa seca da parte aérea (caule+folhas), vagem e raiz do nabo forrageiro, em função da aplicação de torta de filtro e turfa

\begin{tabular}{lcccc}
\hline Tratamento & Dose & caule + folhas & vagem & raiz \\
\cline { 3 - 5 } Turfa & $\mathrm{t} \mathrm{ha}^{-1}$ & & g vaso & -1 \\
\cline { 3 - 5 } & 0 & 10,8 & 4,9 & 0,75 \\
& 20 & 11,7 & 6,2 & 0,73 \\
& 40 & 12,2 & 5,6 & 0,87 \\
& 80 & 12,5 & 5,7 & 0,53 \\
Torta & Médias & 11,8 & 5,6 & $0,72 \mathrm{~B}$ \\
& 0 & 12,1 & 4,8 & 0,9 \\
& 20 & 11,5 & 6 & 0,83 \\
& 40 & 12,6 & 5 & 0,88 \\
& 80 & 12 & 5,9 & 0,97 \\
& Médias & 12,1 & 5,4 & $0,90 \mathrm{~A}$ \\
\hline FV & - & - & - & - \\
Tipo M.O. & - & $\mathrm{ns}$ & $\mathrm{ns}$ & $*$ \\
Dose & - & $\mathrm{ns}$ & $\mathrm{ns}$ & $\mathrm{ns}$ \\
M.O. $x$ Dose & - & $\mathrm{ns}$ & $\mathrm{ns}$ & $\mathrm{ns}$ \\
\hline
\end{tabular}

ANOVA: (ns) não significativo: (*) significativo; médias seguidas teste de Tukey; letras maiúsculas - comparação entre tipos de material orgânico.

grãos de plantas da família Brassicaceae, entre 34 e 58 $\mathrm{mg} \mathrm{kg}^{-1}$ de B (AZARENCO, 2007).

Apesar da alta concentração de B na parte aérea (367 a $646 \mathrm{mg} \mathrm{kg}^{-1}$ ), nas plantas de nabo forrageiro não foram observados sintomas visuais de toxicidade, como folhas queimadas na forma de clorose e necrose (LeE et al., 2008). Lucho-Constantino et al. (2005) reportaram resultados similares, em que várias culturas (vegetais foliares e tubérculos) crescidas em solo com teor de B disponível de 12-14 $\mathrm{mg} \mathrm{dm}{ }^{-3}$, não tiveram sintomas visuais de toxicidade nem diminuição de crescimento. No caso do nabo forrageiro, sua maior tolerância pode estar ligada ao fato de as plantas dicotiledôneas possuírem maior exigência em B, comparadas com as monocotiledôneas (Kabata-Pendias, 2001).

Quando o compartimento da planta analisado foi o caule + folhas, observou-se efeito quadrático pelo uso da torta de filtro (Figura 1), sendo o máximo teor atingido com a dose estimada (derivada primeira da equação) de 33 tha $^{-1}$ de $C$ da torta. Na composição da torta e da turfa, os teores de B estão bem próximos (Tabela 1). Contudo, baseando-se no teor de $C$ dos materiais orgânicos, a adição de B-total foi de $0,97 \mathrm{mg}^{\text {vaso }}{ }^{-1}$ para a torta e de $0,78 \mathrm{mg}$ vaso $^{-1}$ para a turfa, considerando a aplicação de $20 \mathrm{t} \mathrm{ha}^{-1}$. Com a mineralização do material orgânico, houve liberação de $B$, o que se reflete na sua maior de letras diferentes na coluna diferem entre si ao nível de $5 \%$ pelo

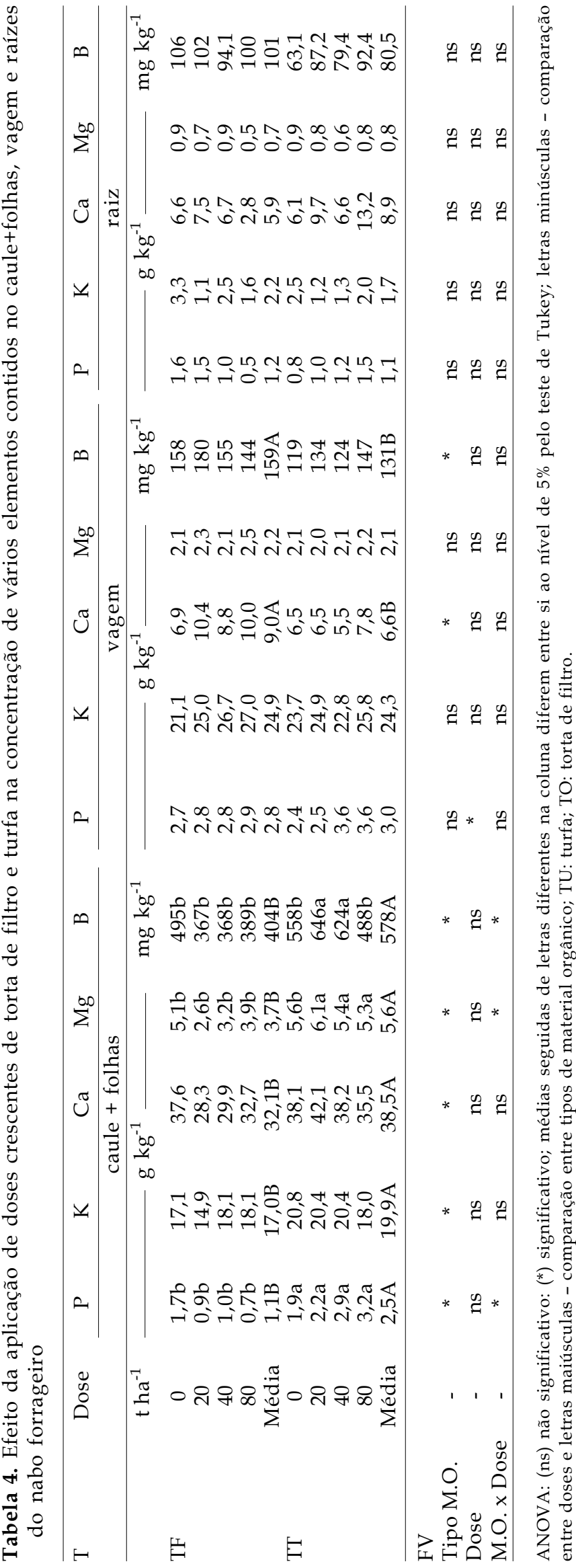

Bragantia, Campinas, v.69, n.2, p.467-476, 2010 


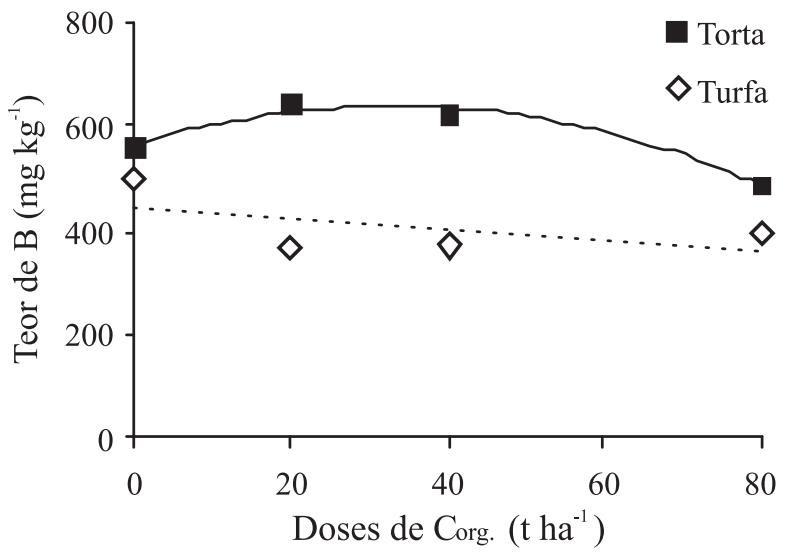

Figura 1. Concentração de B no caule + folha do nabo em função das doses de carbono orgânico aplicadas via torta de filtro $\left(y=-0,069 x^{2}+4,60 x+564 ; r^{2}=0,97 * *\right)$ e turfa $\quad\left(\mathrm{y}=-0,99 \mathrm{x}+439,47 ; \mathrm{r}^{2}=0,30 \mathrm{~ns}\right) ;\left({ }^{* *}\right)$ significativo $(\mathrm{p}<0,05 \%)$; $(\mathrm{ns})$ : não significativo; dose 60 é meramente ilustrativa. Cada símbolo representa o valor médio de três repetições.

disponibilidade no solo, sendo de 8,7 e 7,9 $\mathrm{mg} \mathrm{kg}^{-1}$, respectivamente, para a torta e turfa.

Conforme Basso e Suzuki (2001), o B é um elemento importante para a absorção, transporte e metabolismo de cátions, especialmente o Ca. Contudo, tal efeito não foi observado no presente experimento (Tabela 5). Ainda nesta tabela, verifica-se que o tipo de matéria orgânica adicionada ao solo afetou de maneira diferenciada o acúmulo de Ca e B nas diferentes partes da planta (Tabela 5). No caule + folhas, a torta de filtro proporcionou maior acúmulo de Ca e B; na vagem, o efeito foi significativo para o $\mathrm{Ca}$, com aumento deste elemento em razão da adição de turfa; já nas raízes não houve efeito significativo (Tabela 5).

Outra questão importante refere-se à relação Ca e B na planta em que a amplitude de variação foi de 59,47 a 80,99 mg vaso $^{-1}$ (Tabela 5). AzARenco (2007), trabalhando com alfafa cultivada em solo contaminado com B, sugere como normal um valor de 330 para a relação $\mathrm{Ca} / \mathrm{B}$ e tóxica abaixo de $30 \mathrm{mg}$ vaso $^{-1}$. De acordo com esses valores, a relação $\mathrm{Ca} / \mathrm{B}$, no presente trabalho, estava normal. Esse mesmo autor cita que leguminosas têm maior capacidade de manter a relação $\mathrm{Ca} / \mathrm{B}$ estável, aumentando a resistência da planta a níveis elevados de B no solo. Neste trabalho, o menor valor da relação $\mathrm{Ca} / \mathrm{B}$ foi verificado no tratamento com torta de filtro, sendo de $59,47 \mathrm{mg} \mathrm{vaso}^{-1}$, o qual acumulou no caule + folhas $44 \%$ a mais de $B$ que no tratamento com turfa (Tabela 5). BRENNAM e SHIVE (1948), estudando a interação Ca/B em tecidos vegetais de plantas de tomate, observaram que as plantas que receberam as maiores doses de $\mathrm{B}\left(5 \mathrm{mg} \mathrm{kg}^{-1}\right)$ em combinação com as menores doses de Ca $(5$ e $50 \mathrm{mg} \mathrm{kg}^{-1}$ ), desenvolveram sintomas severos de toxicidade de B. As plantas que receberam a dose de $5 \mathrm{mg} \mathrm{kg}^{-1}$ de B combinada com a dose de $500 \mathrm{mg} \mathrm{kg}^{-1}$ de $\mathrm{Ca}$, não manifestaram os sintomas de toxicidade observados nos outros tratamentos. Esses resultados

Tabela 5. Teor acumulado de B e Ca nas diferentes partes do nabo forrageiro e relação Ca/B na planta toda

\begin{tabular}{|c|c|c|c|c|c|c|c|c|}
\hline \multirow{2}{*}{ Tratamento } & \multirow{2}{*}{ Dose } & $\mathrm{Ca}$ & B & $\mathrm{Ca}$ & B & $\mathrm{Ca}$ & B & \multirow{2}{*}{$\begin{array}{c}\mathrm{Ca} / \mathrm{B} \\
\text { Planta }\end{array}$} \\
\hline & & \multicolumn{2}{|c|}{ caule + folhas } & \multicolumn{2}{|c|}{ vagem } & \multicolumn{2}{|c|}{ raízes } & \\
\hline & $\mathrm{t} \mathrm{ha}^{-1}$ & 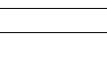 & 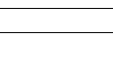 & $\mathrm{m}$ & -1 & & & \\
\hline \multirow[t]{5}{*}{ Turfa } & 0 & 393,8 & 5,27 & 34,61 & 0,80 & 5,04 & 0,08 & 70,43 \\
\hline & 20 & 330,6 & 4,28 & 63,44 & 1,13 & 5,49 & 0,07 & 72,94 \\
\hline & 40 & 362,9 & 4,38 & 51,32 & 0,88 & 5,65 & 0,08 & 78,70 \\
\hline & 80 & 408,8 & 4,89 & 56,29 & 0,82 & 1,53 & 0,05 & 80,99 \\
\hline & Média & $370 \mathrm{~B}$ & $4,7 \mathrm{~B}$ & $51 \mathrm{~A}$ & 0,91 & 4,43 & 0,07 & 74,94 \\
\hline \multirow[t]{5}{*}{ Torta de filtro } & 0 & 411,1 & 5,92 & 32,43 & 0,57 & 4,50 & 0,05 & 68,49 \\
\hline & 20 & 491,6 & 7,55 & 40,62 & 0,84 & 7,30 & 0,06 & 63,82 \\
\hline & 40 & 466,7 & 7,70 & 30,68 & 0,69 & 5,65 & 0,07 & 59,47 \\
\hline & 80 & 433,9 & 5,96 & 44,61 & 0,84 & 6,88 & 0,05 & 70,82 \\
\hline & Média & $450 \mathrm{~A}$ & $6,78 \mathrm{~A}$ & $37 \mathrm{~B}$ & 0,74 & 6,08 & 0,06 & 65,10 \\
\hline \multicolumn{9}{|l|}{$\overline{\mathrm{FV}}$} \\
\hline Tipo M.O. & - & * & * & * & ns & ns & ns & - \\
\hline Dose & - & ns & ns & ns & ns & ns & ns & - \\
\hline M.O. x Dose & - & ns & ns & ns & ns & ns & ns & - \\
\hline
\end{tabular}

ANOVA: (ns) não significativo: $\left(^{*}\right)$ significativo; médias seguidas de letras diferentes na coluna diferem entre si ao nível de $5 \%$ pelo teste de Tukey; letras minúsculas - comparação entre doses e letras maiúsculas - comparação entre tipos de material orgânico. 
Tabela 6. Fator de transferência (F), índice de translocação (IT) e o tempo necessário para remediar o solo contaminado pelo B, usando o nabo forrageiro como planta fitoextratora

\begin{tabular}{lccc}
\hline Tratamento & Fator de Transferência & Índice de Translocação & Tempo de Remoção \\
\hline & & $\%$ & anos \\
Testemunha & 1,19 & 99,3 & 10,6 \\
Turfa & 1,47 & 98,8 & 15,4 \\
Torta & 1,48 & 99,1 & 9,3 \\
\hline
\end{tabular}

sugerem que, em plantas que recebem altas doses de Ca, o excesso de B solúvel é convertido para insolúvel, sendo menos prejudicial à planta.

Esse processo pode ajudar a entender o crescimento normal do nabo forrageiro na presença de altas concentrações de B no solo, mesmo com altas concentrações desse elemento em seus tecidos. Portanto, a calagem feita na área contaminada foi importante para fornecer $\mathrm{Ca}$ às plantas, mantendo um equilíbrio nutricional.

\subsection{Tempo na remoção de $B$ do solo}

A remoção de boro do solo contaminado pelo nabo forrageiro é mostrada na tabela 6 .

O fator de transferência (F) foi muito semelhante entre os materiais orgânicos adicionados, sendo maiores que na testemunha. A adição da torta de filtro ou turfa aumentou em 23\%-24\% a transferência do B do solo para a planta, como era esperado.

O índice de translocação (IT) foi praticamente o mesmo nas três situações avaliadas, com valor médio de 99,1\%, indicando que, apesar de não haver diferenças entre os tratamentos, o B predominou na parte aérea, o que confirma a alta mobilidade desse elemento no xilema, sendo desejável para maior sucesso do processo de fitoextração.

Os valores de F e IT obtidos neste experimento são comparáveis com resultados de plantas hiperacumuladoras que, geralmente, expressam seletividade e acúmulo para certos elementos (Alloway, 1995).

Com relação ao tempo para remoção de metade do B do solo pelo nabo forrageiro, a turfa aumentou o tempo de remoção, ou seja, não atuou favoravelmente no processo de fitoextração em relação ao solo sem receber material orgânico, enquanto a torta de filtro reduziu em 1,3 anos o tempo para a referida remoção (Tabela 5).

Pelos índices apresentados, além das características de planta fitoextratora, o nabo forrageiro é tolerante ao excesso de B. Assim, inferese que seu cultivo na área contaminada tem possibilidade de sucesso pelo uso da fitoextração.

\section{CONCLUSÃO}

A adição de matéria orgânica na forma de torta de filtro é eficiente na redução do tempo necessário para que o nabo forrageiro removesse $50 \%$ do B da área contaminada. A turfa não tem o efeito mitigador de B para o nabo forrageiro. A planta de nabo forrageiro pode ser usada na fitorremediação de solos contaminados com B, sendo eficiente para fitoextração. Tal espécie também tem potencial para revegetação da área, pois possui alta resistência e tolerância nesse ambiente.

\section{REFERÊNCIAS}

ABICHEQUER, A.D.; BOHNEN, H. Eficiência de absorção, translocação e utilização de fósforo por variedades de trigo. Revista Brasileira de Ciência do Solo, v.22, p. 21-26, 1998.

ABREU, C.A.; RAIJ, van B.; ABREU, M.F.; GONZÁLEZ, A.P. Routine soil testing to monitor heavy metals and boron in soils. Scientia Agricola, v.62, p.1-6, 2005.

ABREU, C.A.; ABREU, M.F.; ANDRADE, J.C. Determinação de cobre, ferro, manganês, zinco, cádmio, níquel e chumbo em solos usando a solução de DTPA em pH 7,3 In: RAIJ, van B.; ANDRADE, J.C.; CANTARELLA, H.; QUAGGIO, J.A. (Eds.). Análise química para avaliação da fertilidade de solos tropicais. Campinas: Instituto Agronômico, 2001.

ABREU, M.F. Extração e determinação simultânea por emissão em plasma de nutrientes e elementos tóxicos em amostras de interesse agronômico. 1997. 135p. Tese (Doutorado) Universidade Estadual de Campinas, Campinas.

ALLEONI, L.R.F.; CAMARGO, O.A. Boron adsorption in soils from São Paulo State, Brazil. Pesquisa Agropecuária Brasileira, v.35. p.413-421, 2000.

ALLOWAY, B.J. Heavy metals in soils. London: Blackie Academic, 1995. 368p. 
AZARENKO, Y.A. The boron content in soils of solonetzic complexes in the irtysh region of omsk oblast and the boron resistance of plants. Eurasian Soil Science, v.40, p.512-521, 2007.

BAÑUELOS, G.S.; CARDON, G.; MACKEY, B.; BEN-ASHER, J.; WU, L.; BEUSELINCK,P.; AKOHOUE, S.; ZAMBRZUSKI, S. Boron and selenium removal in boron-laden soils by four sprinkler irrigated plant species. Journal of Environmental Quality, v.22, p.786-792, 1993.

BASSO, C.; SUZUKI, A.; BRAKEMEIER, C. Solos e nutrição. In: EPAGRI (Ed.). Nashi, a pêra japonesa. Florianópolis: Epagri/ Jica, 2001. cap.4, p.139-160. C.

BRAKEMEIER, C. Adubação foliar: a complementação nutricional da macieira. Jornal da Fruta, p.7, 1999.

BISSANI, C.A.; GIANELLO, C.; CAMARGO, F.A.O. ; TEDESCO, M.J. Fertilidade dos solos e manejo da adubação de culturas. 2. ed. Porto Alegre: Metrópole, 2008. 344p.

BOSE, S.; BHATTACHARYYA, A.K. Heavy metal accumulation in wheat plant grown in soil amended with industrial sludge. Chemosphere, v.70, p.1264-1272, 2008.

BRENNAN, E.G.; SHIVE, J.V. Effect of calcium and boron nutrition of the tomato on the relation between these elements in the tissues. Soil Science, v.66, p.65-75, 1948.

BROOKS, R.R. Plants that hyperaccumulate heavy metals: their role in phytoremediation, microbiology, archaeology, mineral exploration and phytomining. Oxford: CAB International, UK, 2004.380p.

CETESB - COMPANHIA DE TECNOLOGIA DE SANEAMENTO AMBIENTAL. Relatório de estabelecimento de valores orientadores para solos e águas subterrâneas para o estado de São Paulo. Disponível em: http:// www.cetesb.sp.gov.br/Solo. Acesso em 22 de fevereiro 2009.

CRUSCIOL, C.A.C.; COTTICA, R.L.; LIMA, E.V.; ANDREOTTI, M.; MORO, E.; MARCON, E. Persistência de palhada e liberação de nutrientes do nabo forrageiro no plantio direto. Pesquisa Agropecuária Brasileira, Brasília, v.40, p.161-168, 2005.

DORDAS, C.; SAH, R.; BROWN, P.H.; ZENG, Q.; HU, H. Remobilização de micronutrientes e elementos tóxicos em plantas superiores. In: FERREIRA, M.E.; CRUZ, M.C.P. RAIJ, B. van; ABREU, C.A. (Ed.). Micronutrientes e elementos tóxicos na agricultura. Jabotical, CNPq/FAPESP/POTAFOS, 2001. p.43-69.

EMBRAPA - EMPRESA BRASILEIRA DE PESQUISA AGROPECUÁRIA. Sistema brasileiro de classificação de solos. Embrapa Solos, 2006. 306 p.

ESPINOZA, W.; OLIVEIRA, A.J. Utilização agrícola dos resíduos da agroindústria canavieira. In: SIMPÓSIO SOBRE FERTILIZANTES NA AGRICULTURA BRASILEIRA, 1984, Brasília. Anais... Brasília, 1984. p.451-470. 1984.

FERREIRA, D.F. Programa SISVAR Versão 5.0 (Build 63). Lavras: UFLA, 1999.

KABATA-PENDIAS, A. Trace elements in soil and plants. 3.ed. Boca Raton, Florida: CRC Press, 2001. 413p.
KEREN, R.; BINGHAM, F.T. Boron in water, soils, and plants. Advanced Soil Science. v.1, p.229-276, 1985.

KOT, F.S. Boron sources, speciation and its potential impact on health. Reviews in Environmental Science and Biotechnology, v.8, p.3-28, 2009.

KUMPIENE, J.; LAGERKVIST, A.; MAURICE, C. Stabilization of $\mathrm{As}, \mathrm{Cr}, \mathrm{Cu}, \mathrm{Pb}$ and $\mathrm{Zn}$ in soil using amendments - A review. Waste Management, v.28, p.215-225, 2008.

LARCHER, W. A utilização dos elementos minerais. In: LARCHER, W. (Ed) Ecofisiologia vegetal. São Carlos: Editora Rima, 2000. cap.3, p.183-230.

LEE, S.B.; LEE, Y.B.; LEE, C.H.; HONG, C.O.; KIM, P.J.; YU, C. Characteristics of boron accumulation by fly ash application in paddy soil. Bioresource Technology, v.99, p.5928-5932, 2008.

LEHTO, T. Boron retention in limed forest mor. Forest Ecology and Management, v.78, p. 11-20, 1995.

LINDSAY, W.L. Inorganic phase equilibria of micronutrients in soil. In: MORTVEDT, J. J.; GIORDANO, P. M.; LINDSAY, W. L. (Eds.). Micronutrients in agriculture. Madison, Soil Science Society of America, 1979. p. 41-57.

LUBBEN, S.; SAUERBECK, D. The uptake and distribution of heavy metals by spring wheat. Water and Air Soil Pollution, v.57-58, p.239-247, 1991.

LUCHO-CONSTANTINO, C.A.; PRIETO-GARCÍA, F.; RAZO, L. M.; RODRÍGUEZ-VÁZQUEZ, R.; POGGI-VARALDO, H.M. Chemical fractionation of boron and heavy metals in soils irrigated with wastewater in central Mexico. Agriculture, Ecosystems and Environment, v.108, p.57-71, 2005.

MARTÍNEZ, M.; BERNAL, P.; ALMELA, C.; VÉLEZ, D.; GARCÍA-AGUSTIIN, P.; SERRANO, R.; NAVARRO-AVIÑÓ, J. An engineered plant that accumulates higher levels of heavy metals than Thlaspi caerulescens, with yields of 100 times more biomass in mine soils. Chemosphere, v.64, p.478-485, 2006.

RAIJ van B.; ANDRADE, J.C.; CANTARELLA, H.; QUAGGIO, J.A (Eds). Análise química para avaliação da fertilidade de solos tropicais. Campinas: Instituto Agronômico, 2001. 285p.

RASKIN, I.; ENSLEY, B.D. Phytoremediation of toxic metals: using plants to clean up the environment. In: BERTI, W.R.; CUNNINGHAM, S.D. Phytostabilization of metals. New York: John Wiley \& Sons, 2000. p.76.

SANTOS. G.A.; SILVA, L.S.; CANNELAS, L.P.; CAMARGO, F.A.O. Fundamentos da matéria orgânica do solo; ecossistemas tropicais \& subtropicais. 2.ed. Porto Alegre: Metrópole, 2008. 654p.

SANTOS, G.C.G.; RODELlA, A.A.; ABREU, C.A. Fitorremediação de solo contaminado por zinco, cobre, manganês, chumbo e boro por plantas de kenaf, mostarda, 
nabo e amaranto. Ciência Rural, 2008.

SCHWAB, P.; ZHU, D.; BANKS, M.K. Heavy metal leaching from mine tailings as affected by organic amendments. Bioresource Technology, v.98, p.2935-2941. 2007.

SHARMA, K.R.; SRIVASTAVA, P.C.;SRIVASTAVA P.; SINGH, V.P. Effect of farmyard manure application on boron adsorption-desorption characteristics of some soils. Chemosphere, v.65, p.769-777, 2006.

SOARES, M.R.; ALLEONI, L.R.F.; CASAGRANDE, J.C. Parâmetros termodinâmicos da reação de adsorção de boro em solos tropicais altamente intemperizados. Química Nova, v.28, p.1014-1022, 2005.

SONNEVELD, C.; DEN ENDEN van J.; DIJK van P.A. Analysis of growing media by means of a 1:1,5 volume extract. Communication in Soil Science and Plants Analysis, v.5, p.183-202, 1974

SYROVETNIK, K. Long-term metal retention processes in a peat bog: field studies, data and modeling. 2005. 86p. Tese (Doutorado). Royal Institute of Technology, Stockholm.

TANAKA, M.; FUJIWARA, T. Physiological roles and transport mechanisms of boron: perspectives from plants. European Journal of Physiology, v.456, p. 671-677, 2008.

USEPA - UNITEDSTATES ENVIRONMENTAL PROTECTION AGENCY. Phytoremediation of contaminated soil and ground water at hazardous waste sites (EPA/540/S-01/500, 2001). Disponível em: http:/ / www.epa.gov Acesso em: 15 de março de 2009.

USEPA - UNITEDSTATES ENVIRONMENTAL PROTECTION AGENCY. Method 3051: microwave assisted acid digestion of sediments, sludges, soil and soils (2007).Disponível em: http://www.epa.gov/epaoswer/hazwaste/test/3_series.htm Acesso em: 10 de janeiro de 2009. 